Chapter 33

\title{
Rhinoplasty in Adult Patients with Cleft - Lip Nasal Deformities
}

\author{
Fereydoun Pourdanesh and Behnam Bohluli \\ Additional information is available at the end of the chapter \\ http://dx.doi.org/10.5772/59237
}

\section{Introduction}

Among facial esthetic surgeons, secondary rhinoplasty in adult cleft -nose deformities is considered to be one of the most challenging surgical interventions due to the congenital distortion of the cartilaginous and bony nasal pyramid, which compromises both nasal esthetics and function. It is generally accepted that the cleft-lip nasal deformity (CLND) is challenging; It is attempted to do this either at the time of primary rhinoplasty in the early of age with repair of lip or by secondary rhinoplasty later on in life. CLND may vary from minor to severe deformity. Several techniques have been suggested and plenty of articles have discussed this issue but still there is no consensus on an optimal technique to manage all of the problems. The common techniques that we use in routine rhinoplasty may not yield good results in patients with CLND, and the reasons for that are:

1. The complexity of anatomical pathology which involves all layers, including skin, cartilage, vestibular lining and skeletal base platform.

2. Numerous former surgical interventions leading to significant scar tissue in the operating site

3. The inevitable effect of growth over time.

The clinical presentation of cleft nose deformities varies widely, requiring a full knowledge of surgical techniques; if deformity is severely asymmetric surgical correction is much more difficult. The clinical features of deformity in a unilateral cleft nose differ from that usually seen in bilateral CLND. The scenario of rhinoplasty in surgical techniques in bilateral CLND is entirely different from unilateral CLND. It seems complete correction of all deficiencies of some noses remain an intangible goal for many, and this is the reason why revision rhinoplasty is commonly needed in these patients. Furthermore, it should be noted that each patient with 
CLND presents a unique challenge due to complexity and combination of aspects and certain techniques that may be more suitable than others in individual cases [1,2].

\section{Primary or secondary rhinoplasty}

Primary rhinoplasty means performing rhinoplasty simultaneously with repair of cleft lip and secondary rhinoplasty means performing rhinoplasty at an early age i.e. during school going age(5-6 years), early adolescence (10-12 years) or later on in life (above 16 yrs. in women and age 18 yrs. in men). But as a working diagnosis, primary rhinoplasty in adults with CLND means the first attempt of surgical intervention on the nose and secondary rhinoplasty means revision rhinoplasty or second operation on the nose.

The best time to attempt correction of CLND is still controversial. With improvement in cleft lip surgery, there is an increasing interest for correction of the nose at the time of lip repair. Some authors strongly recommend a primary rhinoplasty and believe if the procedure is performed correctly it does not adversely affect the growth of the paranasal region. Primary rhinoplasty improves nasal symmetry in patients with unilateral cleft lip deformity. This does not exclude the possibility of later revisional surgery although there is tendency to doing an appropriate primary repair of cleft lip deformity but the fact is, small defects that are left after primary repair are amplified with the growth process and affect adjacent structures. It is become clear that primary and secondary rhinoplasty at the same time of lip repair or at age 7-8 can lead to some kind of deformities in adulthood. The adult deformity is related not only to the original embryological mesodermal deficiency and diminished growth potential, but also to the pattern of primary surgery, the degree of interceptive surgery during growth and the level of orthodontic skill practiced within a particular treatment. In our center most cases are referred for primary rhinoplasty in adulthood [3-9].

\section{Clinical signs and symptoms}

A variety of clinical signs may be seen in an operated cleft lip and palate patient as well as in unoperated cases. We are usually faced with a wide variety of signs and symptoms in a repaired cleft lip case with or without primary rhinoplasty. In this section the clinical signs of cleft nose deformity will be discussed.

\subsection{Unilateral cleft lip nose deformity}

Clinical features of cleft nose deformity from a cosmetic point of view have varied from minor to severe (Figure 1).

It is not easy to accurately describe the anatomic pathology of secondary CLND. Components of the nasal deformity include defects of all layers of skin, cartilages, septum, entire nasal pyramid as well as hypoplasia and mal positioning of the maxillary segments and the anatomic 

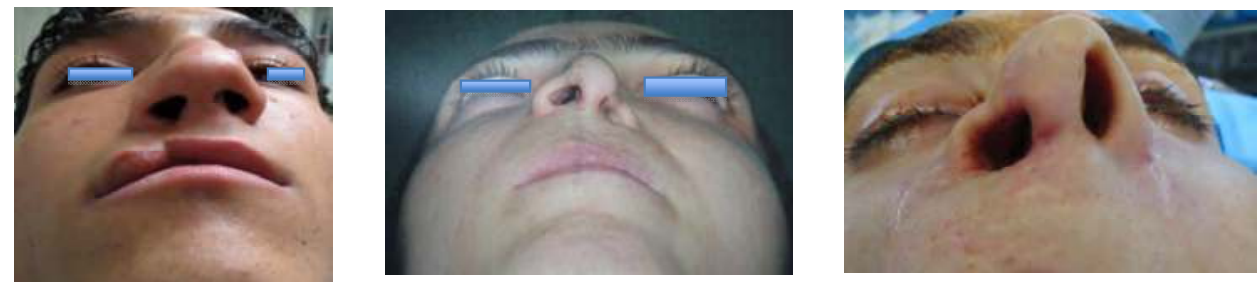

Figure 1. Basal view of minor and two difficult cases of unilateral cleft nose.

and functional deformity of the orbicularis muscle. It is accepted that patients who undergo appropriate primary repair for cleft lip will have secondary deformities [8]. Recently many three dimensional studies have been performed on cleft lip nose deformity patients; thanks to advances in technology we can define the details of the anatomic and functional deformity of each component [10]. The cleft deformity is not restricted to the skin and cartilage. Hogan represented the unilateral cleft nasal deformity as a tilted tripod (Figure 2).
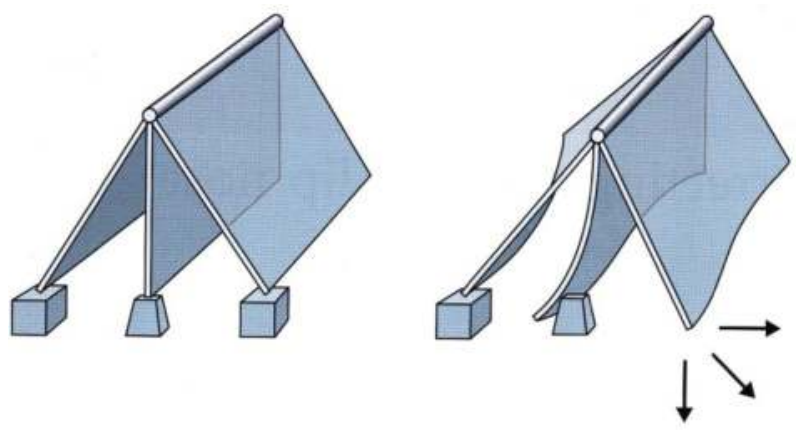

Figure 2. Tilted nasal tripod in CLND

Because of the lack of skeletal support, the alar base on the cleft side can, in some cases, become retro positioned with growth, even following an appropriate primary correction [9]. In most cases however good the primary correction, the patient is left with asymmetry of the nasal base and nares [11].

The ala on the cleft side is lengthened vertically and lies below the alae on the non- cleft side. The lower lateral cartilage is depressed and spread across the cleft. The nasal tip is deviated toward the left side. The columella on the left side is shortened significantly, as compared with the non-cleft side. The columella is obliquely oriented. With its base deviated to and located in the non-cleft side away from the midline. Bilateral alar bases are asymmetric, with the cleft side alar base inferiorly and posteriorly displaced. [12]

The deformities such as a deficient tubercle, vermilion deficiency, irregularities, the short upper lip, long upper lip, tight upper lip, and unfavorable scars may be common seen in the 
repaired lip [9]. Also the severity of septal deformity is variable. Typically, the septum is dislocated from the maxillary crest towards the non-cleft side resulting in a septal deflection towards the cleft side commonly causing nasal obstruction on that side. In addition, the inferior turbinate on the cleft side is also frequently hypertrophic, further adding to nasal obstruction on that side. [13] Lee [8] described seven cardinal deformities in unilateral cleft lip nose deformity include:

- Caudal deflection of the nasal septum to the non-cleft side

- Deviation of the nasal dorsum

- Low setting of the medial crus

- Tethering deformity of the lateral crus

- Discontinuity of the orbicularis oris muscle

- Long or short lip deformity

- Absence of the philtral column

Although Huffman and Lierle in 1949 published the most detailed descriptions of the cleft lip nasal deformity, over time it has changed; the typical clinical features of the unilateral cleft nasal deformity (Figure 3) is characterized as follow:
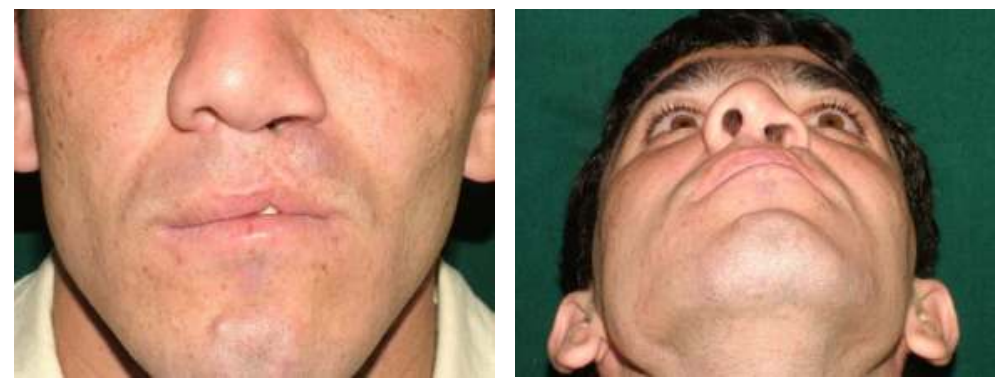

Figure 3. Typical deformity in unilateral cleft nose deformity

1. The tip of the nose and caudal septum are deviated towards the non-cleft side.

2. The base of the columella also deviates towards the non-cleft side.

3. The convexity of the septum on the side of the cleft impinges on the airway.

4. The angle between the medial and lateral crura on the cleft side is excessively obtuse.

5. The dome of the alar cartilage on the cleft side is depressed.

6. The interior of the cleft-side nostril, from its apex down the cephalic margin of the alar cartilage to the pyriform aperture, is bowed by a linear contracture-the vestibular web.

7. The lateral crus is caudally displaced on the cleft side. 
8. The cleft side ala buckles inwardly (M configuration)

9. The cartilage on the cleft side smaller than the normal side

10. Absent alar-facial groove on the cleft side

11. Hypoplastic maxilla on cleft side

12. Ill-proportioned nares

13. Widened nostril floor on cleft side

14. Retrodisplaced medial crus on the cleft-side

15. Oblique slant of the columella [8-14]

\subsection{Bilateral cleft nose deformities}

Adult patients with bilateral cleft lip nose deformity (BLCND) show some asymmetry. Typical characteristics of BLCND include: a short columella, a short, depressed, thick nasal tip, flatappearing nasal tip, sometimes notched in the midline, large diverging nostrils, wide nostril floors, an obtuse columella-labial angle, wide alae, a short nasal bridge, and a wide nasal root, lateral displacement of both alar domes with bilateral dislocation of the lateral crura from the septum, hooding of the alar rims and flaring alar bases. The short columella is the most common problem in bilateral cleft nose deformity. Although the residual deformity in bilateral cleft is symmetrical correction of nasal tip widening, the retro- positioning of the alar cartilage and shortening of the columella are all encountered and difficult to treat. The secondary deformity involves the nose and the lip as well as the facial profile (Figure 4) [12, 15, and 16].

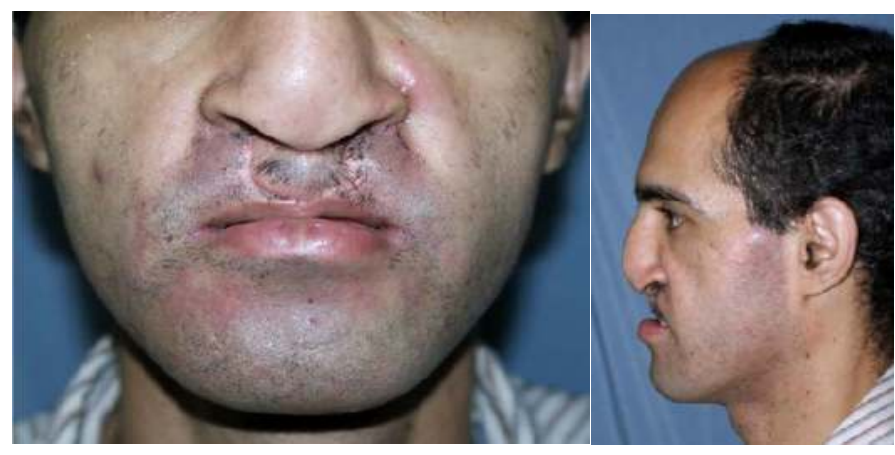

Figure 4. Preoperative views A: Frontal view B: lateral view

\section{Diagnosis}

In order to treat CLND, clinical diagnosis and complete knowledge of anatomy, pathology and physiology of the nasal pyramid, maxilla, and lip is imperative. Clinical examination 
consists of a careful examination of the bony and cartilaginous skeleton, anterior rhinoscopy evaluating the appearance of the nasal mucosa and the position of the anterior part of the septum is necessary. The problems present in a patient with a cleft lip nasal deformity must be recognized just as any other rhinoplasty patient and clearly defined in order to formulate a successful treatment plan. In fully grown adult we need to evaluate:

1. The nose and lip

2. Midface deficiency

3. Oro- nasal fistula

4. Occlusion and

5. Speech.

Significant improvement in growth, function and esthetics has been achieved by almost normal reconstruction of alveolar clefts.To establish the nasal skeletal base, three dimensional reconstruction of alveolar defects with bone grafting has been advocated by clinicians; different approaches at the different stages of life have been suggested (Figure 4).
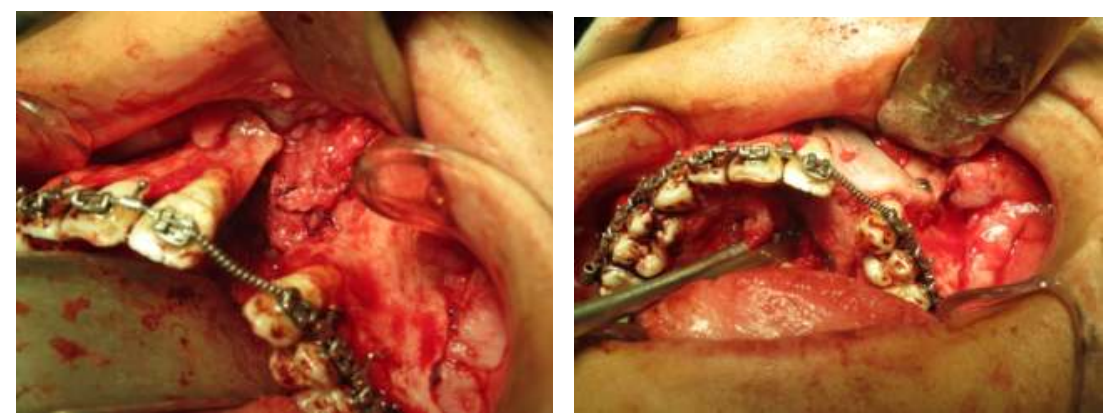

Figure 5. Intraoperative view of 3D reconstruction of alveolar cleft

Proper jaw relationship plays an important role in the skeletal base of the nose; obviously, final rhinoplasty must be postponed until completion of orthognathic surgeries. Although some authors suggested performing orthognathic surgery on growing cleft patients when mandated by psychological and / or functional concerns; but, because of postsurgical outcome the consensus of most clinicians is to delay orthognathic surgery until growth is completed. Different kinds of distraction procedures such as intraoral and extraoral devices may be used as an alternative to the orthognathic approaches in cleft patients; a proper position of the maxilla is mandatory before performing rhinoplasty in adults with cleft lip and nose deformity. Supposedly, the skeletal support enhances the projection of the lip and nose on the cleft side.

CT scan of paranasal sinuses in axial and coronal views may be helpful to define the deformation of the septum as well as other intranasal structures. It is important to identify both aesthetic and functional problems associated with the cleft nose deformity. Each component 
of the deformity must be addressed in an orderly manner including the skeletal base, nasal dorsal bone and cartilage, nasal tip cartilage, and, finally, the skin envelope [1, 8, 11, 17-21].

\section{Non-surgical treatment}

Before correction of the soft tissues, it is important to make sure if there are any dental problems that needs to be corrected first. Soft tissue correction before dental treatment can result in very embarrassing situation that may not be correctable later [22].

\section{Surgical treatment}

No single procedure has given sufficiently satisfactory results to provide a surgical standard for CLND correction. Despite considerable progress in the treatment of patients with cleft lip and palate, there is still no agreement about the optimal treatment method. Secondary deformity after the primary operation is a significant problem encountered in cleft-lip repair [23]. The knowledge and experience of the surgeon in rhinoplasty is the keystone for correcting deformity. Use of four basic techniques in rhinoplasty such as onlay grafting, suturing methods, cartilage transection, reorientation and cartilage repositioning can help the cosmetic surgeon to overcome many of the problems inherent to these patients. Familiarity with the numerous techniques in this regard and selection of the proper one to treat the existing deformity is essential. No matter which technique is used it is important to address all parts of the deformity and set all parts in anatomic position. Nostril asymmetry is one of the main complaints of adult patient with unilateral cleft nose deformities. In 1977, Tajima and Maruyama introduced an operation in which the deformed alar cartilage was fixed to the upper lateral cartilage through a reverse- $\mathrm{U}$ incision, and the insufficient area within the nostril was filled with the overhanging alar web tissues. We use this method for the correction of severe asymmetric nostrils. To obtain ideal treatment outcomes in unilateral cleft nose deformity the below list of procedures are used.

1. Using open rhinoplasty approach

2. Doing septoplasty and harvesting graft from the septum via open approach incision.

3. Release septum to upper lateral cartilage on the non-cleft side.

4. Release upper lateral cartilage from the skin and nasal pyriform

5. Correcting deformed caudal and dorsal part of the septum which is fixed to the non-cleft side.

6. Release and splitting of lower lateral cartilage on the cleft side.

7. Medial and lateral crus elevation

8. Using reverse $U$ incision for correcting web. 
9. Using Z- plasty incision in vestibular pica for vertical lengthening.

10. Reshaping and repositioning of lower lateral cartilage is essential for correcting nostril deformity.

11. Using multiple sutures to stabilize the final shape of lower and upper lateral cartilages.

12. Using autogenous graft for augmentation

13. Augmentation approach is better than reduction

14. Strut, tip, sheen and batten grafts are help to restore the nasal tip.

15. Unequal alar base resection

16. Release depressor septi muscles

Internal or external osteotomy can be used (Figure 5) [8, 24].
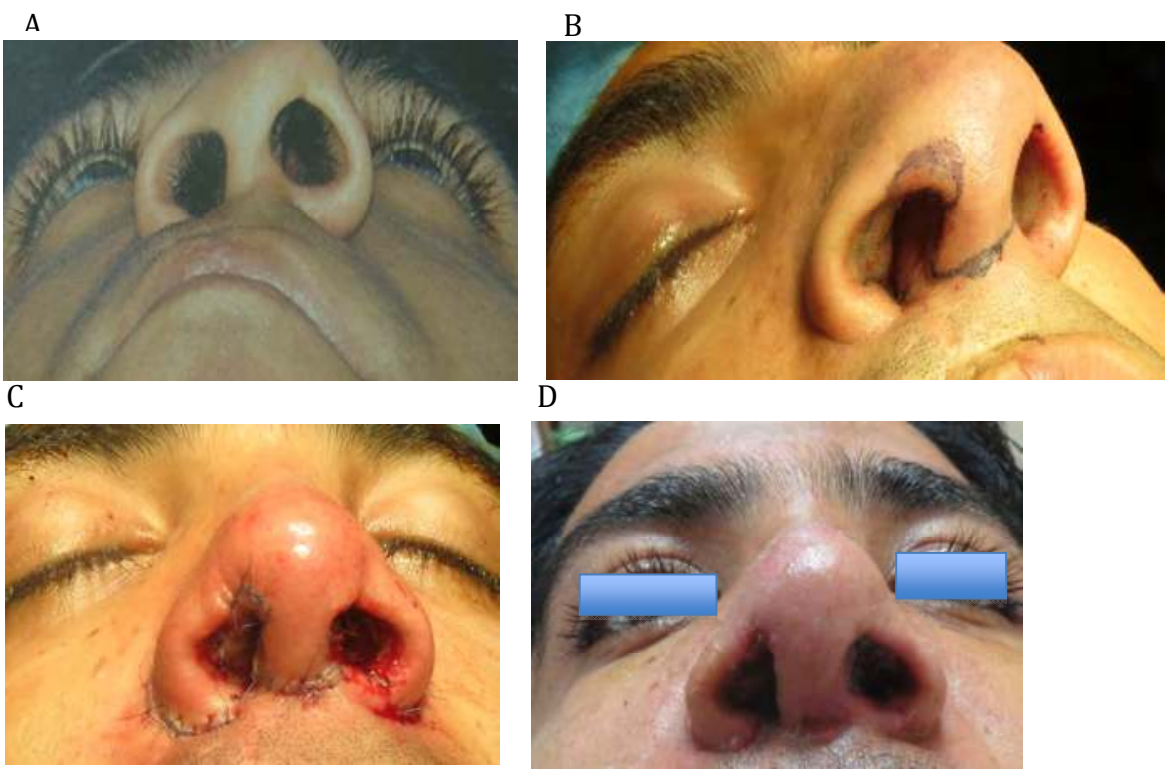

D

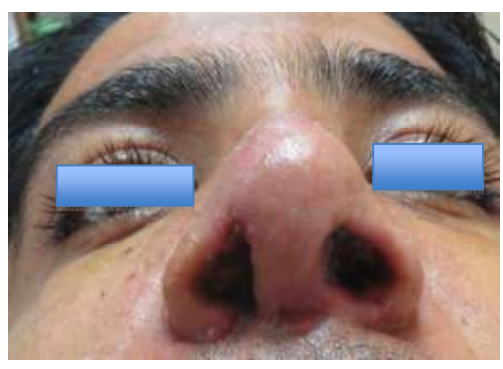

Figure 6. A, Before surgery: B, Intraoperative: C, Immediately after surgery: D, one week post surgery

In bilateral cleft nose deformity although confronted with almost symmetric deformities the lengthening of the columella, correction of the depressed nasal tip, bilateral dislocation of alar cartilage and eversion of the alar bases are on the top of the clinician's concern. There are many ways to elongate the shortness of the columella such as forked flap, v-y advancement, prolabium advancement flap combined with an Abbe flap, composite graft and skin rim rotation flap [26]. Using strong and proper struts, repositioning and reshaping lower lateral cartilages, supraperiosteal dissection of the pyriform area to allow the reposition of nasal 
correcting alar component, use of different suturing methods and augmentation with autogenous grafts can help to achieve almost ideal results (Figure 6).

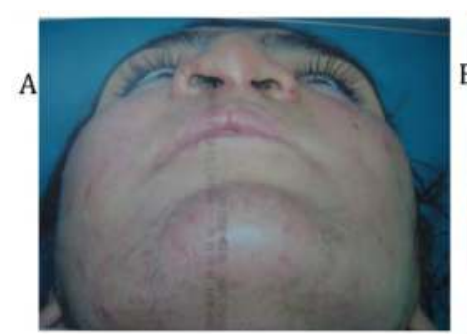

D

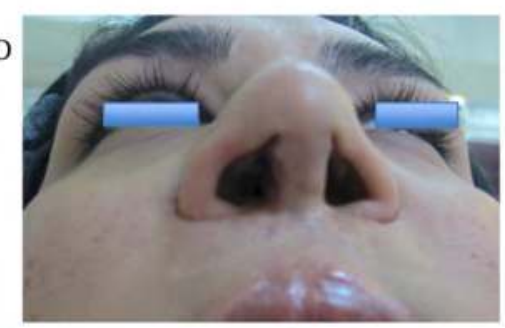

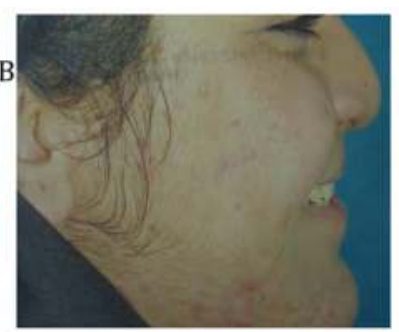

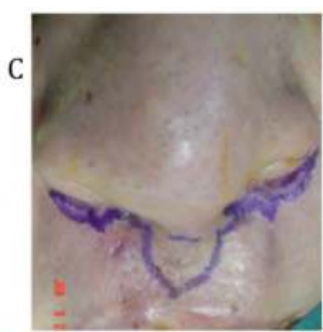

E

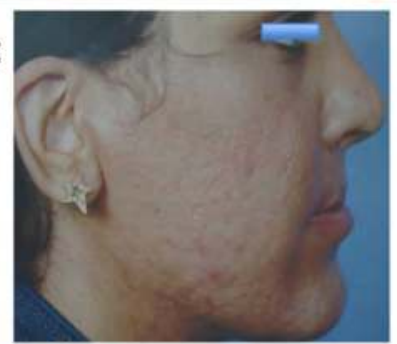

Figure 7. A and B, before surgery basal and lateral view, C: intraoperative D and E: 6 months after surgery

\section{Outcomes}

Normalized esthetics of the lip and nose is on the top of the specific goals of surgical care for children born with cleft lip and palate followed by nasal airway patency and normal speech [25]. The focus of secondary correction of unilateral cleft-lip nose deformity has been nasal symmetry. Importance has been placed on correction of the cleft-lip nasal deformity by translocation of the alar cartilage with its attached vestibular lining into a normal position, thereby establishing the normal vault and shape of the cartilage.[23] There is no doubt that definitive rhinoplasty should logically only be undertaken after reconstruction of skeletal base and correction of the jaws relationship. The key point is overcorrecting the cleft-side nostril and its alar cartilage is believed to produce better symmetry compensating for possible relapse during the postoperative period [12]

\section{Complications}

There is no difference between the complications associated with cleft lip rhinoplasty and traditional open rhinoplasty in non-cleft patients. Theoretically the risk of infection is more likely particularly when cartilage grafts are used. The risk of bleeding is similar to that of 
traditional rhinoplasty. Patients should be warned of possible need for secondary rhinoplasty, need for minor or even major revision, existence of nostril asymmetry, visible scars, skin necrosis, dysfunction of nasal system and anomalies associated with the donor site. Clinical failures using different kinds of grafts consisting of autograft, allograft and alloplastic materials may be seen in the future. Failure to achieve the desired nasal contour and normal looking appearance is a common finding in cleft lip nasal rhinoplasty.

\section{Author details}

Fereydoun Pourdanesh ${ }^{1^{*}}$ and Behnam Bohluli ${ }^{2}$

*Address all correspondence to: drpourdanesh@gmail.com

1 Associate Professor, Department of Oral and Maxillofacial Surgery, School of Dentistry, Shahid Beheshti University of Medical Sciences, Tehran, Iran

2 Associate Professor, Department of Oral and Maxillofacial Surgery, School of Dentistry, Azad University, Tehran, Iran

\section{References}

[1] Hellings P et al, Unique approach to secondary cleft lip rhinoplasty in facial plastic surgery, B-ENT, 2010,6:97-101

[2] Monasterio, O,F Ruas J,E, Cleft Lip Rhinoplasty: The Role of Bone and Cartilage grafts clinics in Plastic Surgery-Vol. 16, No. 1, January 1989,177-187

[3] Anastassov E.G,Joos U., Comprehensive management of cleft lip and palate deformities, Joms, Volume 59, Issue 9, 2001, Pages 1062-1075

[4] Thomas C, Primary Rhinoplasty for the Cleft Nasal Deformity. J Def Manag 2013 S3: 005. Doi:10.4172/2167-0374.S3-005

[5] Penfold C,Douminguez - gonzalez S Bilateral cleft lip and nose repair,bjomfs. Volume 49, Issue 3, 2011, Pages 165-17

[6] Nakajima T, Ogata H, Sakuma H, Long-term outcome of simultaneous repair of bilateral cleft lip and nose (a 15 year experience),brithis journal of plastic surgry,2003, (56),205-217

[7] El bestar M, Mansour O: Cleft lip nasal deformity : primary repair,Egypt.J,plast. Reconstr.surg.vol 28 No, 1 2004, pages 15-21 
[8] Lee DW, Choi B-K, Park Be-Y.Y, Seven Fundamental Procedures for Definitive Correction of Unilateral Secondary Cleft Lip Nasal Deformity in Soft Tissue Aspects, J Oral Maxillofac Surg,2011 :69:e420-e430,

[9] BANKS P, the surgical anatomy of secondary cleft lip and palate deformity and its significance in reconstruction, british journal of oral surgery (1983) 21, 78-93

[10] Nakamura N, Okawachi, T, Nozoe E,, Nishihara K, and Matsunaga K, Three-Dimensional Analyses of Nasal Forms After Secondary Treatment of Bilateral cleftlip-Nose Deformity in Comparison to Those of Healthy Young Adults, J Oral Maxillofac Surg 69:e469-e481, 2011

[11] Hopper A.R, Cutting C, Grason B, chapter 23, cleft lip and palate,Grabb and smith's plastic surgery, $6^{\text {th }}$ edition by Charles $H$ Thorne,2007, pages 201-225

[12] Banks p, the surgical anatomy of secondary cleft lip and palate deformity and its significance in reconstruction, British Journal of Oral Surgery (1983) 21, 78-93

[13] Lun-Jou Lo, Primary Correction of the Unilateral Cleft Lip Nasal Deformity: Achieving the Excellence, Chang Gung Med J 2006;29:262-7)

[14] Huffman WC,Leirle DM,studies on the pathologic anatomy of the unilateral harelip nose, plast reconstr surg,1949,4:225

[15] Yonehara, Y, Mori Y, Chikazu D, Saijo H, Tsuyoshi Takato T, Secondary Correction of Bilateral Cleft Lip and Nasal Deformity by Simultaneous Placement of an Abbe Flap, Septal Cartilage Graft, and Cantilevered Iliac Bone Graft, J Oral Maxillofac Surg 66:581-588, 2008

[16] Ahmed SA, Ramadan M.M, Correction of Secondary Deformities of the Cleft Lip Nose,Suez Canal Univ Med J Vol. 11, No. 1, March, 20081 -7

[17] Precious S.D, A New Reliable Method for Alveolar Bone Grafting at About 6 Years of Age, J Oral Maxillofac Surg 67:2045-2053, 2009

[18] Epker N.B, Correction of the Skeletal Nasal Base in Rhinoplasty, J Oral Maxillofac Surg 49:939-946, 1991

[19] Pourdanesh f.Behnia H, Using l-shaped bone graft to restore alar base region in patients with alveolar cleft, International Journal of Oral \& Maxillofacial Surgery Volume 42, Issue 10, Page 1202, October 2013

[20] Wolford M.L, Cassano S.D, Cottrell A.D, El Deeb M, Karras, Goncalves R.J, Orthognathic Surgery in the Young Cleft Patient: Preliminary Study on Subsequent Facial Growth, J Oral Maxillofac Surg 66:2524-2536, 2008

[21] Showkatbakhsh R, Pourdanesh F, Jamilian A, Ghorbani A, Behnaz M. Hyrax application as a tooth-borne distractor for maxillary advancement., J Craniofac Surg. 2011 Jul;22(4):1361-6. 
[22] Tung K.M, Management of Post Cleft Deformities of the Lip \& Nose,medical bulletin,vol.12,no 11,November 2007

[23] Fujimoto T, Imai K, Hatano T, Makoto Takahashi, Tamai M, Follow-up of unilateral cleft-lip nose deformity after secondary repair with a modified reverse-U method, Journal of Plastic, Reconstructive \& Aesthetic Surgery (2011) 64, 747e753

[24] Tajima S, Maruyama M: Reverse-U incision for secondary repair of cleft lip nose. Plast Reconstr Surg 60:256, 1977

[25] Taheri Talesh K, Kalantar Motamedi MH, cleft lip and palate surgery, A text book of advanced oral and maxillofacial surgery p 560- 569

[26] Nakamura N, Sasaguri M, Okawachi T, Nishihara K, Nozoe E, Secondary correction of bilateral cleft lip nose deformity e Clinical and three-dimensional observations on pre- and postoperative outcome, Journal of Cranio-Maxillo-Facial Surgery 39 (2011) $305 \mathrm{e} 312$ 